Comment. Math. Helv. 76 (2001) 300-313

(C) 2001 Birkhäuser Verlag, Basel

0010-2571/01/020300-14\$1.50+0.20/0

Commentarii Mathematici Helvetici

\title{
Hyperbolic 3-manifolds as cyclic branched coverings
}

\author{
Marco Reni and Bruno Zimmermann \\ Marco Reni sadly died in an accident in the mountains in June 2000
}

\begin{abstract}
There is an extensive literature on the characterization of knots in the 3-sphere which have the same 3 -manifold as a common $n$-fold cyclic branched covering, for some integer $n \geq 2$. In the present paper, we study the following more general situation. Given two integers $m$ and $n$, how are knots $K_{1}$ and $K_{2}$ related such that the $m$-fold cyclic branched covering of $K_{1}$ coincides with the $n$-fold cyclic branched covering of $K_{2}$. Or, seen from the point of view of 3-manifolds: in how many different ways can a given 3-manifold occur as a cyclic branched covering of knots in $S^{3}$. Under certain hypotheses, we solve this problem for the basic class of hyperbolic 3-manifolds and hyperbolic knots (the other basic class is that of Seifert fiber spaces resp. of torus and Montesinos knots for which the situation is well understood; the general case can then be analyzed using the equivariant sphere and torus decomposition into Seifert fiber spaces and hyperbolic manifolds).
\end{abstract}

Mathematics Subject Classification (2000). 57M12, 57M25, 57M50.

Keywords. Hyperbolic knot, cyclic branched covering, hyperbolic 3-manifold.

\section{Introduction}

The cyclic branched coverings of knots and links in the 3 -sphere constitute an important class of 3-manifolds, and the reciprocal determination of knots and links and their cyclic branched coverings has been a theme of constant interest in lowdimensional topology. In particular, it has been studied extensively the question when two different knots can have the same $n$-fold cyclic branched covering. In the present paper, we study the following more general problem: given two integers $m$ and $n$, how are knots $K_{1}$ and $K_{2}$ related such that the $m$-fold cyclic branched covering of $K_{1}$ and the $n$-fold cyclic branched covering of $K_{2}$ coincide; or, seen from the point of view of 3-manifolds: in how many different ways can a given 3 -manifold occur as a cyclic branched covering of knots in $S^{3}$. We solve this problem, under certain hypotheses, for the basic class of hyperbolic 3-manifolds and hyperbolic knots. We note that the other basic class is that of Seifert fiber spaces; here, by results of Meeks and Scott ([MS]), we can assume that the covering groups are fiber-preserving, so we are dealing with the cases of torus knots and 
Montesinos knots for which the situation is well understood in terms of Seifert invariants. The general case can then be analyzed using the equivariant sphere theorem and torus decomposition ([MS]) into Seifert fiber spaces and hyperbolic manifolds.

The paper is a continuation of work started in [R1] and [Z1] where the case of homology 3-spheres resp. the case $m=n$ have been studied.

Before stating our results, we describe two basic constructions of examples.

\subsection{Standard abelian construction I}

Let $m$ and $n$ be positive integers, and let $L=\bar{X} \cup \bar{Y}$ be a 2-component link in the 3 -sphere such that both components $\bar{X}$ and $\bar{Y}$ are trivial knots. We suppose that the linking number of $\bar{X}$ and $\bar{Y}$ is relatively prime to both $m$ and $n$. Then the $n$-fold cyclic branched covering of $\bar{Y}$ (that is of $S^{3}$ along $\bar{Y}$ ) is the 3 -sphere, and the preimage $X$ of $\bar{X}$ is connected and hence a knot. Similarly, we get a knot $Y$ in $S^{3}$ as the preimage of $\bar{Y}$ in the $m$-fold cyclic branched covering of $\bar{X}$. Now the $m$-fold cyclic branched covering of $X$ and the $n$-fold cyclic branched covering of $Y$ is the same 3 -manifold $M$ which is the regular branched $\mathbb{Z}_{m} \times \mathbb{Z}_{n}$-covering of the link $L$ (i.e. with covering group $\mathbb{Z}_{m} \times \mathbb{Z}_{n}$ ).

It has been shown in [Z1] that, in case $n=m$ and if $n$ is not a power of two, that this is the only way how to construct different hyperbolic knots with the same $n$-fold cyclic branched covering. For $n=2$ there are other ways of construction and the situation is more complicated; a solution in this case has been given in [R2]. In the present paper, we consider the case $n \neq m$ which is more difficult and requires different methods; nevertheless, under certain hypotheses we will obtain similar results. Basically, in the case $m=n$ (or if $m$ and $n$ have a common prime divisor $p$ ), the situation is determined by a single Sylow $p$-subgroup of the isometry group of the cyclic branched covering (a hyperbolic 3-manifold in this paper) whereas when $m$ and $n$ are coprime one has to relate Sylow subgroups for different primes. In any case our methods use in a crucial way results from finite group theory.

\subsection{Standard abelian construction II}

Let $l, m$ and $n$ be three different integers which are pairwise coprime. Let $L=$ $X_{0} \cup Y_{0} \cup Z_{0}$ be a link of three trivial (unknotted) components such that any two components of $L$ form a Hopf link (in particular they have linking number one).

The $n$-fold cyclic branched covering of $Z_{0}$ is the 3 -sphere, and the preimages $X_{1}$ of $X_{0}$ and $Y_{1}$ of $Y_{0}$ form a link $X_{1} \cup Y_{1}$ of two trivial components of linking number $n$. The preimage of $X_{1}$ in the $m$-fold cyclic branched covering of $Y_{1}$ (which is again the 3 -sphere) is a knot $X$ in $S^{3}$. Finally, the $l$-fold cyclic branched covering of $X$ is a 3-manifold $M$ which, by construction, is also the regular branched $\left(\mathbb{Z}_{l} \times \mathbb{Z}_{m} \times \mathbb{Z}_{n}\right)$ covering of the link $L$ (i.e. with covering group $\mathbb{Z}_{l} \times \mathbb{Z}_{m} \times \mathbb{Z}_{n} \cong \mathbb{Z}_{l m n}$ ). 
Now, by cyclically permuting the roles of the components $X_{0}, Y_{0}$ and $Z_{0}$ of $L$, we get three knots $X, Y$ and $Z$ in $S^{3}$ such that $M$ is the $l$-fold cyclic branched covering of $X$, the $m$-fold cyclic branched covering of $Y$ and the $n$-fold cyclic branched covering of $Z$.

Note that a similar construction works also for links $L$ with more than three components (and also for integers which are not coprime) but that one inevitably ends up with links with more than one component then.

A nice example of a manifold $M$ arising from construction 0.2 is the Poincaré homology 3 -sphere which is the 5 -fold cyclic branched covering of the $(2,3)$-torus knot (the trefoil), the 3 -fold cyclic branched covering of the $(2,5)$-torus knot and the 2-fold cyclic branched covering of the $(3,5)$-torus knot. In this case the 3 component link $L$ is the (3,3)-torus link, with branching orders 2,3 and 5 associated to its three components; more generally, by associating (coprime) branching orders $l, m$ and $n$ to its components, one obtaines the Brieskorn homology sphere of type $(l, m, n)$. The Poincaré homology sphere is spherical. Explicit hyperbolic examples for construction 0.2 can be obtained as in section 4.1 of [RZ2], see in particular the proof of Theorem 4; as the construction is quite long we do not repeat it here.

An interesting example of a manifold arising from construction 0.1 is the hyperbolic Matveev-Fomenko-Weeks manifold of smallest known volume which is obtained by associating branching orders 2 and 3 to the two components of the (symmetric) link $7_{1}^{2}$. This manifold is the 2 -fold cyclic branched covering of the knot $9_{49}$ and the 3 -fold cyclic branched covering of the knot $5_{2}$ (see [MV]).

A knot or link $K$ in the 3 -sphere is hyperbolic if its complement $S^{3}-K$ admits a complete hyperbolic structure (Riemannian metric of constant negative curvature) of finite volume. For an integer $n \geq 2$, it is $2 \pi / n$-hyperbolic if the 3 -sphere admits a hyperbolic metric which becomes singular, with an angle of $2 \pi / n$, around the components of the link. If we denote by $\mathcal{O}_{n}(K)$ the 3 -orbifold whose underlying topological space is the 3 -sphere and whose singular set, of branching index $n$, is the knot $K$, another way of saying this is that $\mathcal{O}_{n}(K)$ is a hyperbolic 3-orbifold; equivalently, the $n$-fold cyclic branched covering of $K$ is a hyperbolic 3-manifold and the cyclic covering group acts by isometries. It is a consequence of the orbifold geometrization theorem ([T1], [T2],[BP]) that every hyperbolic knot is also $2 \pi / n$ hyperbolic, for $n>3$, and for $n=3$ the only exception is the figure- 8 knot which is $2 \pi / 3$-euclidean (see [Z1] for a discussion of the relation between these classes of knots).

In the following, $M$ will be always a closed hyperbolic 3-manifold.

Theorem 1. Let $M$ be the $n$-fold and $m$-fold cyclic branched covering of inequivalent hyperbolic knots $K$ and $K^{\prime}$, respectively. Suppose that $m$ and $n$ have a common prime divisor $p>2$. Then $K$ and $K^{\prime}$ arise from the standard abelian construction 0.1. Moreover there are at most two such knots. 
We note that $K$ and $K^{\prime}$ are inequivalent if $m$ and $n$ are different. This is because, for a fixed knot $K$, the volumes of the hyperbolic 3-orbifolds $\mathcal{O}_{n}(K)$ increase monotonously with $n$ (see e.g. $[\mathrm{K}]$ ), so different cyclic branched coverings of the same knot $K$ have different volumes.

The basic case of 2-fold branched coverings has been analyzed in [R2] for knots and in [RZ2] for links, and it turns out that the situation for $p=2$ is more complicated.

In particular, Theorem 1 gives a short proof of the following result from [Z1].

Corollary. Let $M$ be the $n$-fold cyclic branched covering of inequivalent hyperbolic knots $K$ and $K^{\prime}$ such that $n$ is not a power of two. Then $K$ and $K^{\prime}$ arise from the standard abelian construction 0.1. Moreover there are at most two such knots.

A cyclic symmetry of a knot $K$ is a periodic diffeomorphism of $\left(S^{3}, K\right)$ with nonempty fixed point set $F$ disjoint from $K(F$ is an unknotted circle by the positive solution of the Smith conjecture). The quotient is again the 3 -sphere, and $K$ and $F$ project to a 2-component link $\bar{K} \cup \bar{F}$. We call a 2-component link symmetric if there exists an orientation-preserving diffeomorphism of $S^{3}$ which exchanges its two components.

Theorem 2. Let $M$ be the $n$-fold and $m$-fold cyclic branched covering of inequivalent hyperbolic knots $K$ and $K^{\prime}$, respectively, such that $m$ and $n$ are not powers of two. Suppose that

i) $K$ is not strongly invertible;

ii) $K$ has no cyclic symmetry of order $n$ such that the associated quotient link $\bar{K} \cup \bar{F}$ is symmetric.

Then $K$ and $K^{\prime}$ arise from the standard abelian construction 0.1.

Theorem 3. Under the hypotheses of Theorem 2, there are at most three inequivalent hyperbolic knots in $S^{3}$ having $M$ as a cyclic branched covering of branching orders which are not powers of two. If there are three different such knots then they arise from the standard abelian construction 0.2.

In [RZ1] sets of four different $\pi$-hyperbolic knots have been constructed with the same 2-fold cyclic branched covering. It is shown in [R2] that there are at most nine such knots, but for the moment four is the maximal number known for such an example.

If $n$ is a power of a prime $p$ then the $n$-fold cyclic branched covering of $K$ is a $\mathbb{Z}_{p}$-homology sphere (see e.g. [G]). Hence if $M$ is not a $\mathbb{Z}_{2}$-homology sphere Theorem 3 is best possible.

Basically the proof of Theorem 2 works also if $n$ or $m$ is a power of two, but in these cases there are various other standard constructions and the situation becomes more technical. For $n=2$, the simplest situation after the standard abelian 
construction is a standard dihedral construction where the covering involution of $K$ acts as a strong inversion on the fixed point set of the covering group of $K^{\prime}$ (so $K^{\prime}$ is strongly invertible); examples can be constructed by equivariant surgery on suitable knots with dihedral symmetry. If $n$ or $m$ is a power of two there are still other standard situations. All these various constellations can be summarized algebraically in a standard solvable situation saying that a Sylow $p$-subgroup $S_{p}$ of the orientation-preserving isometry group $G$ of $M$ normalizes some Sylow $q$-subgroup $S_{q}$ of $G$ (see the proof of Theorem 2); here $p$ and $q$ are different prime numbers such that $p$ divides $n$ but not $m$, and viceversa. Note that, by a Sylow theorem, up to conjugation we can assume that the corresponding Sylow subgroups of the cyclic covering groups of $K$ and $K^{\prime}$ are subgroups of $S_{p}$ and $S_{q}$, respectively, so $S_{p}$ and $S_{q}$ determine completely the geometry of the situation. For odd primes $p$, we will show in Lemma 1 that the Sylow $p$-subgroups of the isometry group $G$ of $M$ are abelian of rank one or two. Under the hypotheses i) and ii) of Theorem 2, this remains true also for $p=2$; in general, the Sylow 2-subgroup of a 2-fold branched covering contains such an abelian group of rank $\leq 2$ as a subgroup of index $\leq 4$, see $[\mathrm{R} 2]$.

So Theorem 2 can be generalized for branching orders which are powers of two. On the other hand, it seems that the condition of non-invertibility of $K$ is essential for Theorem 2. In this regard we note that our results remain true for cyclic branched coverings of knots in arbitrary homology 3 -spheres; in this more general case, as the Smith conjecture is no longer true, there may occur a new abelian situation where the two covering groups have the same fixed point set. Examples of hyperbolic homology 3 -spheres admitting actions of the dodecahedral group $A_{5} \cong \operatorname{PSL}(2,5)$ with two global fixed points are easily constructed (for example by equivariant surgery on a link in $S^{3}$ with $A_{5}$-symmetry, taking one component of the link for each edge of the dodecahedron and a "Borromean type" constellation at each vertex in order to have trivial linking numbers). The quotients of such a homology 3 -sphere by the elements of orders 2,3 and 5 of $A_{5}$ are again homology 3spheres, and the branch sets are knots. It is clear then that, for branching orders 3 and 5 , we are not in a standard abelian or solvable situation (the branching order 2, in combination with 3 or 5 , gives an example of a standard dihedral construction). In particular, Theorem 2 does not remain true for cyclic branched coverings of invertible knots in arbitrary homology 3 -spheres, but at moment we do not have such examples for the 3 -sphere itself.

We note that a knot which does not satisfy condition ii) is very special, so this is not really a strong restriction.

For our next results we adopt a group theoretical point of view. For the case of solvable groups we have the following complete solution.

Theorem 4. Let $M$ be the $n$-fold and $m$-fold cyclic branched covering of inequivalent hyperbolic knots $K$ and $K^{\prime}$, respectively, such that $m$ and $n$ are not powers of two. Suppose that the isometry group of $M$ is solvable. Then $K$ and $K^{\prime}$ arise 
from the standard abelian construction 0.1.

If $n$ is even we can reduce the situation to the solvable case and thus to the standard abelian situation; note that the condition of non-invertibility is no longer needed here.

Theorem 5. Let $n$ be an even integer greater or equal to six. Let $M$ be the $n$-fold cyclic branched covering of a hyperbolic knot $K$ such that condition ii) of Theorem 2 holds. Then the isometry group of $M$ is solvable.

We note that Theorem 5 remains valid also for $n=2$ and $n=4$ if $K$ satisfies both conditions i) and ii) of Theorem 2 (in fact the proof of Theorem 2 applies for $p=2$ showing that $G$ is a semidirect product $S_{2} U$ where $S_{2}$ is a Sylow 2-subgroup of $G$ and the normal subgroup $U$ has odd order and is thus solvable).

By Theorems 2 and 4 we are left with the basic case in which the isometry group $G$ of $M$ is non-solvable and the knot $K$ is invertible; in particular, $G$ contains an involution with connected fixed point set (a lift of a strong inversion). It can be shown that in this case the isometry group of $M$ is of a very restricted type. In contrast to the previous theorems, the methods from finite group theory which we have to use here are quite involved so we do not follow this line here.

\section{Proof of Theorem 1}

Since $K$ and $K^{\prime}$ are hyperbolic, they are also $2 \pi / n$ - and $2 \pi / m$-hyperbolic because $n$ and $m$ are greater than two (here we exclude branching order three for the figure- 8 knot; in fact the 3 -fold cyclic branched covering of the figure- 8 knot is the euclidean Hantzsche-Wendt manifold which does not occur, however, as a cyclic branched covering of any other knot, see [Z2]). So in the rest of the paper we will always assume that the covering manifold $M$ is hyperbolic and that the covering groups act by isometries.

We need the following

Lemma 1. Let $M$ be a hyperbolic 3-manifold which is the $n$-fold cyclic branched covering of a hyperbolic knot $K$, and let $p$ be an odd prime dividing $n$. Then a Sylow p-subgroup of the orientation-preserving isometry group of $M$ is either cyclic or a direct product of two cyclic groups. Moreover there are exactly one or two simple closed curves in $M$ which are fixed by some nontrivial element in $S_{p}$ with connected fixed point set (and consequently each of these curves is invariant under the action of $S_{p}$ ).

Proof of Lemma 1. Let $G$ denote the finite orientation-preserving isometry group of the hyperbolic 3-manifold $M, S_{p}$ a Sylow $p$-subgroup of $G, H \subset G$ the cyclic 
covering group of $K$ and $H_{p}$ the Sylow $p$-subgroup of $H$. Up to conjugation, we can assume that $H_{p}$ is a subgroup of $S_{p}$.

The fixed point set of the groups $H_{p} \subset H$ is the preimage $\tilde{K}$ of $K$ in $M$. Let $A:=N_{S_{p}} H_{p}$ be the normalizer of $H_{p}$ in $S_{p}$. Then $A$ maps the fixed point set $\tilde{K}$ of $H_{p}$ to itself; because $p>2, A$ acts as group of rotations around and along $\tilde{K}$ (but not as reflections). It follows that $A$ is cyclic or a direct product of two cyclic groups.

Suppose that $A$ is cyclic. Then, if an element of $S_{p}$ normalizes $A$, it normalizes also $H_{p}$, hence $N_{S_{p}} A=A$. Now the normalizer of a proper subgroup of a finite $p$-group is strictly larger than the subgroup ([Su1, p.88, Theorem 1.6]). Thus $A=S_{p}$. Moreover $\tilde{K}$ is the only simple closed curve fixed pointwise by some nontrivial element of $S_{p}$ (because this is the case for $H_{p}$ ).

Now suppose that $A$ is a direct product of two cyclic groups. Note that $A$ commutes with $H$ (because it maps the fixed point set $\tilde{K}$ of $H$ to itself), and hence also $H$ maps the fixed point set of each element of $A$ to itself. Moreover $A$ projects to $\left(S^{3}, K\right)$. By the positive solution of the Smith conjecture ([MB]), the projection $\bar{A}$ of $A$ is cyclic (acting as rotations along $K$ ), and either $\bar{A}$ acts freely or there is exactly one simple closed curve in $S^{3}$ occuring as the fixed point set of elements of $\bar{A}$. It follows that besides $\tilde{K}$ there is at most one other simple closed curve in $M$ which is fixed by some nontrivial element of $A$ with connected fixed point set (such a curve is invariant under the covering group $H$, so different curves project to different curves in $\left(S^{3}, K\right)$ ).

It follows that each element in the normalizer $N_{S_{p}} A$ maps $\tilde{K}$ to itself (because $p$ is odd) and hence normalizes $H_{p}$. Thus again $N_{S_{p}} A=A$ and consequently $A=S_{p}$.

This finishes the proof of Lemma 1.

Remark. In the proof of Lemma 1, the solution of the Smith conjecture can be avoided using instead (the elementary) Lemma 3 in [RZ2] wich states that a group $\mathbb{Z}_{p} \times \mathbb{Z}_{p}$ of diffeomorphisms of $S^{3}$ (or of any homology 3-sphere) has exactly two different subgroups $\mathbb{Z}_{p}$ with nonempty (connected) fixed point set.

Proof of Theorem 1. As before, let $S_{p}$ be the Sylow $p$-subgroup of the orientationpreserving isometry group $G$ of $M, H$ and $H^{\prime}$ the covering groups of $K$ and $K^{\prime}$, and $H_{p}$ and $H_{p}^{\prime}$ the Sylow $p$-subgroups of $H$ and $H^{\prime}$. Up to conjugation, we can assume that $H_{p}$ and $H_{p}^{\prime}$ are subgroups of $S_{p}$. By Lemma $1, S_{p}$ is cyclic or a direct product of two cyclic groups.

If $S_{p}$ is cyclic then there is a unique simple closed curve which is fixed by nontrivial elements of $S_{p}$. Thus the preimages $\tilde{K}$ and $\tilde{K}^{\prime}$ of $K$ and $K^{\prime}$ coincide, hence $H$ and $H^{\prime}$ commute (having common fixed point set $\tilde{K}=\tilde{K}^{\prime}$ ) and project to $\left(S^{3}, K^{\prime}\right)$ and $\left(S^{3}, K\right)$, respectively. By the solution of the Smith conjecture, this can happen only if $H=H^{\prime}$. Then $K=K^{\prime}$ that is $K$ and $K^{\prime}$ are equivalent.

Now suppose that $S_{p}$ is a direct product of two cyclic groups and that $K$ and 
$K^{\prime}$ are not equivalent. As before, the Smith conjecture implies that $\tilde{K}$ and $\tilde{K}^{\prime}$ are distinct. As $H_{p}$ and $H_{p}^{\prime}$ commute, also $H$ and $H_{p}^{\prime}$ commute (mapping the fixed point set $\tilde{K}$ of $H$ to itself), and finally also $H$ and $H^{\prime}$ commute (mapping the fixed point set $\tilde{K}^{\prime}$ of $H^{\prime}$ to itself). Thus we are in the standard situation 0.1. By Lemma 1 , there are exactly two simple closed curves fixed by some nontrivial element of $S_{p}$ (which are $\tilde{K}$ and $\tilde{K}^{\prime}$ ). This implies that there are only two inequivalent knots having $M$ as a cyclic branched covering with branching orders divided by $p$.

This finishes the proof of Theorem 1 .

The proof implies the following lemma which will be used later.

Lemma 2. In the situation of Theorem 1, the Sylow p-subgroup $S_{p}$ of $G$ is a direct product of two cyclic groups, and $\tilde{K}$ and $\tilde{K}^{\prime}$ are the only simple closed curves which are fixed by some nontrivial element of $S_{p}$.

\section{Proof of Theorem 2}

Crucial for the proof is the following theorem of Burnside, see [Su2, p.143, Theorem 2.10].

Proposition 1. Let $G$ be a finite group and $S_{p}$ a Sylow p-subgroup of $G$, for a prime $p$. Then if $S_{p}$ lies in the center of its normalizer $N_{G} S_{p}$ in $G$ (in particular $S_{p}$ is abelian), $G$ splits as a semidirect product $U S_{p}$ of $S_{p}$ and a characteristic subgroup $U$ of $G$.

Proof of Theorem 2. If there is an odd prime dividing $m$ and $n$ then Theorem 1 applies, therefore we can assume that there are distinct odd primes $p$ and $q$ dividing $n$ and $m$, respectively. We use the notation at the begining of the proof of Lemma 1. Also, $H^{\prime} \subset G$ denotes the cyclic covering group of $K^{\prime}, S_{q}$ a Sylow $q$-subgroup of $G$ and $H_{q}^{\prime}$ a Sylow $q$-subgroup of $H^{\prime}$. We can assume that $H_{p} \subset S_{p}$ and $H_{q}^{\prime} \subset S_{q}$.

In order to apply Proposition 1, we will show first that $S_{p}$ lies in the center of its normalizer $N_{G} S_{p}$ in $G$, in both cases of Lemma 1 . By Lemma 1, there are exactly one or two simple closed curves in $M$ which are fixed by some nontrivial element of $S_{p}$ with connected fixed point set. Because $p$ is odd, each of these curves is invariant under $S_{p}$.

If there is exactly one such curve then this is $\tilde{K}$ and consequently each $g \in N_{G} S_{p}$ maps $\tilde{K}$ to itself and normalizes $H_{p}$. Then $g$ normalizes also $H$ and thus projects to $\left(S^{3}, K\right)$. Now $g$ can act as a rotation around or along $\tilde{K}$ or as a reflection on $\tilde{K}$. In the first case $g$ commutes with $S_{p}$. In the second case $g$ has order two, the fixed point set of $g$ meets $\tilde{K}$ in exactly two points, and consequently $g$ projects to a strong inversion of $K$ which was excluded by condition i). Thus $S_{p}$ lies in the 
center of its normalizer in $G$ and Proposition 1 applies.

Now suppose that there is exactly one other simple closed curve $\tilde{F}$ in $M$ fixed pointwise by some nontrivial element of $S_{p}$ with connected fixed point set. Each $g \in N_{G} S_{p}$ maps $\tilde{K} \cup \tilde{F}$ to itself. Suppose that $g$ maps $\tilde{K}$ to itself. As in the first case, $g$ cannot act as a reflection on $\tilde{K}$; thus it acts as a rotation and hence commutes with every element of $S_{p}$. If $g$ exchanges $\tilde{K}$ and $\tilde{F}$ then $g H g^{-1}$ fixes $\tilde{F}$ pointwise, commutes with $H$ and projects to a cyclic symmetry of order $n$ of $K$. Now we are exactly in the situation which was excluded by condition ii). Thus again $S_{p}$ lies in the center of its normalizer.

By Proposition 1, there exists a normal subgroup $U$ of $G$ such that $G$ splits as a semidirect product $G=S_{p} U$. As $p \neq q$, any Sylow $q$-subgroup of $G$, in particular $S_{q}$, lies in $U$. The group $S_{p}$ acts by conjugation on the set of Sylow $q$-subgroups of $U$; by one of the Sylow theorems, the number of elements of this set divides the order of $U$. The number of elements of each orbit of this action is a power $p^{\alpha}$ of $p$, where $\alpha \geq 0$ (the stabilizer or normalizer of an element of an orbit is a subgroup of $S_{p}$ ). As $p$ does not divide the order of $U$, it follows that some orbit has only one element. Thus $S_{p}$ normalizes a Sylow $q$-subgroup of $U$. As all Sylow $q$-subgroups are conjugate in $U$ we can assume that $S_{p}$ normalizes $S_{q}$, and we have a semidirect product $S_{p} S_{q}$ as a subgroup of $G$.

Now Lemma 1 applies also to the Sylow $q$-subgroup $S_{q}$ of $G$. As $p$ is odd and $S_{p}$ normalizes $S_{q}$ it follows that the preimage $\tilde{K}^{\prime}$ of $K^{\prime}$ is invariant under $S_{p}$. Both $S_{p}$ and $S_{q}$ act as rotations around and along $\tilde{K}^{\prime}$ and hence commute elementwise (in particular, the above semidirect product of $S_{p}$ and $S_{q}$ is really a direct product). Moreover $H_{p}$ (in fact $S_{p}$ ) commutes also with $H^{\prime}$ which implies that $\tilde{K}$ is invariant under $H^{\prime}$ and, finally, that $H$ and $H^{\prime}$ commute. Note that $\tilde{K}^{\prime}$ is different from $\tilde{K}$, by the positive solution of the Smith conjecture, in particular $H$ and $H^{\prime}$ have trivial intersection. Thus we have a subgroup $H \times H^{\prime}$ of $G$. This is the covering group of a 2-component link in $S^{3}$, and hence we are in the standard situation 0.1.

This finishes the proof of Theorem 2 .

The proof gives also the following result which will be needed later.

Lemma 3. Let $p$ and $q$ be different odd prime numbers such that $p$ divides $n$ but not $m$, and $q$ divides $m$ but not $n$. Then, under the hypotheses of Theorem 2, the Sylow subgroups $S_{p}$ and $S_{q}$ of $G$ are cyclic and commute. Moreover, also $K^{\prime}$ satisfies conditions i) and ii) of Theorem 2.

Proof. By the proof of Theorem 2, $S_{p}$ and $S_{q}$ commute and $\tilde{K}^{\prime}$ is invariant under $S_{p}$. By Lemma $1, S_{p}$ is cyclic or a direct product of two cyclic groups. If $S_{p}$ is a direct product of two cyclic groups then $\tilde{K}^{\prime}$ is fixed pointwise by some nontrivial element $g$ of $S_{p}$. Now $g$ commutes also with the covering group $H^{\prime}$ of $K^{\prime}$ and hence projects to a nontrivial periodic diffeomorphism of $S^{3}$, contradicting the solution of the Smith conjecture. So this case does not occur and $S_{p}$ is cyclic. 
Finally, $\tilde{K}^{\prime}$ is not strongly invertible because a strong inversion of $\tilde{K}^{\prime}$ would be also a strong inversion of $\tilde{K}$, and $K$ and $K^{\prime}$ do not have cyclic symmetries of orders $p$ and $q$, respectively.

\section{Proof of Theorem 3}

Suppose that $M$ is the $n_{i}$-fold cyclic branched covering of inequivalent hyperbolic, so $2 \pi / n_{i}$-hyperbolic, knots $K_{i}, i=0, \ldots, r$, where $K=K_{0}, n=n_{0}$ and the $n_{i}$ are not powers of two.

Suppose there is an odd prime $p$ dividing $n=n_{0}$ and some $n_{i}, i>0$. By the last part of Theorem 1, $p$ does not divide any other $n_{i}$. By Lemma $2, S_{p}$ is a product of two cyclic groups. Thus the situation of Lemma 3 does not occur which implies that there are only two different knots, i.e. $r=1$ (because by hypothesis no $n_{i}$ is a power of two).

Thus we can assume that any two different $n_{i}$ have no common prime divisor different from two. Let $p_{i}$ be distinct odd primes dividing $n_{i}$. Let $S_{p_{i}}$ be a Sylow $p_{i}$-subgroup of $G$. We may assume that the Sylow $p_{i}$-subgroup $H_{p_{i}}$ of the covering group $H_{i}$ of $K_{i}$ is a subgroup of $S_{p_{i}}$. By the solution of the Smith conjecture, the fixed point sets $\tilde{K}_{i}$ of the $H_{i}$ are all distinct. By Lemma 3 , all $S_{p_{i}}$ are cyclic and commute. Then also all $H_{i}$ commute, and we have a subgroup $H_{0} \times \ldots \times H_{r}$ of $G$. In particular, each $\tilde{K}_{i}$ is invariant under each $H_{j}$. The projection of the cyclic group $S_{p_{1}} \times \ldots \times S_{p_{r}}$ to $\left(S^{3}, K_{0}\right)$ is a cyclic group $A$ of diffeomorphisms of $S^{3}$ whose fixed point set consists of at least $r$ simple closed curves occuring as fixed point sets of nontrivial elements of $A$; these are the projections $\bar{K}_{1}, \ldots, \bar{K}_{r}$ of $\tilde{K}_{1}, \ldots, \tilde{K}_{r}$.

By the solution of the Smith conjecture, $\bar{K}_{1}$ is unknotted. The complement of the interior of a regular neighbourhood of $\bar{K}_{1}$ is a solid torus on which the cyclic group $A$ acts by restriction. Such an action is known to be a standard rotation (using e.g. the equivariant Dehn Lemma) and has at most one component of fixed point sets (the central curve), because $A$ does not have order two. It follows that $r$ is at most two.

If $r=2$ we have a subgroup $H_{0} \times H_{1} \times H_{2}$ of $G$ which is the covering group of a 3-component link $L$ in $S^{3}$. The components of $L$ are trivial by the Smith conjecture, and the induced branched covering of each two of them is the 3-sphere. By [Sa], the Hopf link is the only link in $S^{3}$, other than the trivial knot, which has the 3 -sphere as an abelian branched covering. Thus each two components of $L$ form a Hopf link and we are in the situation of the standard construction 0.2.

\section{Proof of Theorem 4}

By Theorem 1, we can assume that there exist distinct odd prime divisors $p$ and $q$ of $n$ and $m$, respectively. As before, we denote by $S_{p}$ and $S_{q}$ a Sylow $p$-subgroup 
resp. a Sylow $q$-subgroup of $G$, of orders $p^{a}$ resp. $q^{b}$. By the generalization of the Sylow theorems for solvable groups ([Su2, p.104, Theorem 5.6]), there exists a subgroup $G_{0}$ of order $p^{a} q^{b}$ of $G$, and we can assume, up to conjugation, that $S_{p}$ and $S_{q}$ are subgroups of $G_{0}$. By Lemma 1 , each of $S_{p}$ and $S_{q}$ is cyclic or a direct product of two cyclic groups, and in particular abelian. As $G_{0}$ does not contain elements of even order, the second part of Lemma 1 implies that $S_{p}$ lies in the center of its normalizer in $G_{0}$. By Proposition $1, G_{0}$ splits as a semidirect product $U S_{p}$, for a normal subgroup $U$ of $G_{0}$. By conjugation, we can assume that $U=S_{q}$. Thus $S_{p}$ normalizes $S_{q}$. As in the proof of Theorem 1, this implies that $S_{p}$ and $S_{q}$ commute elementwise (hence $U=S_{p} \oplus S_{q}$ ). Thus we are in the standard situation 0.1 and the Theorem is proved.

\section{Proof of Theorem 5}

Crucial for the proof is the following theorem of Glauberman, see [Su2, p.315, Theorem 2.14].

$Z^{*}$-Theorem. Let $h$ be an involution in a finite group $G$ and $S_{2}$ a Sylow 2subgroup of $G$ containing $h$. Denote by $\mathcal{O}(G)$ the maximal normal subgroup of odd order of $G$. Then the following are equivalent:

i) $h$ is not conjugate in $G$ to an element in $S_{2}$ different from $h$;

ii) the image of $h$ is central in the quotient group $G / \mathcal{O}(G)$.

We also need to describe the local action of a group of diffeomorphisms in the neighboorhood of a simple closed curve. More precisely, let $G$ be a finite group of orientation-preserving diffeomorphisms of a closed orientable 3-manifold and suppose that $G$ contains an element, say $h$, with nonempty connected fixed point set $\tilde{K}$. Any element $g$ of $G$ which commutes with $h$ maps the fixed point set $\tilde{K}$ of $h$ to itself. Therefore $g$ induces a reflection (strong inversion) or a rotation on $\tilde{K}$ : we shall call such elements $h$-reflections, respectively $h$-rotations (a nontrivial element which acts trivially on $\tilde{K}$ will also be a $h$-rotation).

So the centralizer $C_{G} h$ of $h$ in $G$ is the set of $h$-rotations and $h$-reflections: the subgroup of $h$-rotations is a normal subgroup of $C_{G} h$ of index one or two. We have

Proposition 2. Let $G$ be a finite group of orientation-preserving diffeomorphisms of a closed orientable 3-manifold; suppose that $G$ contains an element $h$ with nonempty connected fixed point set. Then the centralizer $C_{G} h$ of $h$ in $G$ is isomorphic to a subgroup of a semidirect product $\mathbb{Z}_{2}\left(\mathbb{Z}_{a} \times \mathbb{Z}_{b}\right)$, for some nonnegative integers $a$ and $b$, where $\mathbb{Z}_{2}$ operates on the normal subgroup $\mathbb{Z}_{a} \times \mathbb{Z}_{b}$ (the subgroup of h-rotations) by sending each element to its inverse. 
Proof of Theorem 5. Since $n$ is even, the covering group $H$ of $K$ contains an involution $h$. Let $S_{2}$ be a Sylow 2-subgroup of $G$ containing $h$. In order to apply the $Z^{*}$-Theorem, we will prove the following

Claim. $h$ is not conjugate in $G$ to any element in $S_{2}$ different from $h$.

Assuming the Claim for the moment, we will show now that $G$ is solvable. The $Z^{*}$-Theorem implies that the image $\bar{h}$ of $h$ is central in $G / \mathcal{O}(G)$. By a Sylow theorem, all involutions in the group generated by $h$ and the normal subgroup of odd order $\mathcal{O}(G)$ are conjugate to $h$, by an element of $\mathcal{O}(G)$ as we may assume. This implies easily, using that each element of $G / \mathcal{O}(G)$ commutes with $\bar{h}$, that $G / \mathcal{O}(G)$ is the surjective image of the centralizer $C_{G} h$ of $h$ in $G$. It follows from Proposition 2 that $C_{G} h$ is solvable, therefore also $G / \mathcal{O}(G)$ is solvable. Since also the group $\mathcal{O}(G)$, of odd order, is solvable by the Feit-Thompson Theorem, it follows that $G$ is solvable and Theorem 5 is proved.

Proof of the Claim. It is enough to prove that $h$ is not conjugate in $G$ to an element of $C_{S_{2}} h$ different from $h$. Then, if $S_{2}$ coincides with $C_{S_{2}} h$, this gives the Claim. Suppose that $C_{S_{2}} h$ is a proper subgroup of $S_{2}$. By [Su1, p.88, Theorem 1.6] the normalizer of any proper subgroup of a nilpotent group is strictly larger than the subgroup. Let $g$ be an element in $S_{2}$ which normalizes $C_{S_{2}} h$ but is not in $C_{S_{2}} h$. Then $g$ does not commute with $h$ and conjugates $h$ to an element of $C_{S_{2}} h$ different from $h$. This is a contradiction, so this case does not occur.

So it remains to show that $h$ is not conjugate in $G$ to an element of $C_{S_{2}} h$ different from $h$. Suppose, by contradiction, that there exists a conjugate $g h g^{-1}$ different from $h$ in $C_{S_{2}} h$, for some $g \in G$. The fixed point set of $h$ and $H$ is the preimage $\tilde{K}$ of $K$ in $M$. As $h$ and $g h g^{-1}$ commute, $h$ maps the fixed point set $g(\tilde{K})$ of $g h g^{-1}$ and of $g H^{-1}$ to itself and acts either as a rotation or as a reflection on $g(\tilde{K})$. We will show that both cases do not occur. Note that $g(\tilde{K})$ is different from $\tilde{K}$ (because $g h g^{-1}$ is different from $h$ ).

If $h$ acts as a reflection on $g(\tilde{K})$ then $\tilde{K}$ and $g(\tilde{K})$ intersect in exactly two points. Each of these intersection points is fixed by the distinct cyclic groups $H$ and $\mathrm{gHg}^{-1}$, of order $n$, which are thus contained in the isotropy group of the point. This isotropy group is a finite subgroup of the orthogonal group $S O(3)$. By hypothesis, $n \geq 6$, and there are no such finite subgroups of $S O(3)$ (the only finite subgroups of $S O(3)$ containing elements of order greater or equal to six are cyclic or dihedral). So this case does not occur.

Suppose now that $h$ acts as a rotation on $g(\tilde{K})$. Then the fixed point sets $\tilde{K}$ and $g(\tilde{K})$ are disjoint, and the groups $H$ and $g H^{-1}$ commute generating a group $H \oplus g H g^{-1}$ whose elements act as rotations on both $\tilde{K}$ and $g(\tilde{K})$. We note that $\tilde{K}$ and $g(\tilde{K})$ are exactly the two simple closed curves in $M$ which are fixed by some nontrivial element of $H \oplus g H^{-1}$ (the projection of $H \oplus g H g^{-1}$ to $S^{3}=M / H$ is cyclic and has the projection of $g(\tilde{K})$ as fixed point set; by classical Smith fixed 
point theory, there can occur no other components of fixed points for nontrivial elements).

Also the group $g \mathrm{Hg}^{-1} \oplus g^{2} \mathrm{Hg}^{-2}$ acts by rotations on $g(\tilde{K})$ which implies that $g^{2} \mathrm{Hg}^{-2}$ is a subgroup of $H \oplus g \mathrm{Hg}^{-1}$ (because the latter group contains already all rotations of order $n$ of $g(\tilde{K}))$. Then the fixed point set $g^{2}(\tilde{K})$ of $g^{2} H^{-2}$ has to be equal to $\tilde{K}$, so $g^{2}(\tilde{K})=\tilde{K}$ and $g^{2} H g^{-2}=H$. It follows that $g$ normalizes $H \oplus g H g^{-1}$ and thus projects to the quotient $M /\left(H \oplus g H g^{-1}\right)$. But this gives exactly the situation ii) in Theorem 2 which is excluded in Theorem 5 (with $\bar{F}$ as the projection of $g(\tilde{K}))$. So also the second case does not occur.

This finishes the proof of the Claim and of Theorem 5 .

\section{References}

[BP] M. Boileau, J. Porti, Geometrization of 3-orbifolds of cyclic type. Prépublication 119, Laboratoire de Math. E. Picard, Toulouse 1998.

[G] C. McA. Gordon, Some aspects of classical knot theory. Knot Theory. Proceedings, Plans-sur-Bex, Switzerland (J. C. Hausmann, ed.) Lect. Notes Math. 685, SpringerVerlag, 1977, 1-60.

[K] S. Kojima, Hyperbolic 3-manifolds singular along knots. Chaos, Solitons and Fractals Vol. 9, No. 45, 765-777 (1998).

[MB] J. Morgan, H. Bass, The Smith Conjecture. Academic Press, New York 1984.

[MS] W. H. Meeks, P. Scott, Finite group actions on 3-manifolds. Invent. Math. 86 (1986), 287-346.

[MV] A. Mednykh, A. Vesnin, Covering properties of small volume hyperbolic 3-manifolds. J. Knot Theory Ramific. 7 (1998), 381-392.

[R1] M. Reni, Finite group actions with fixed points on homology 3-spheres. Mh. Math. 128 (1999), 23-33.

[R2] M. Reni, On $\pi$-hyperbolic knots with the same 2-fold branched covering. Math. Ann. 316 (2000), 681-697.

[RZ1] M. Reni, B. Zimmermann, Isometry groups of hyperbolic 3-manifolds which are cyclic branched coverings. Geom. Dedicata 74 (1999), 23-35.

[RZ2] M. Reni, B. Zimmermann, On hyperelliptic involutions of hyperbolic 3-manifolds. To appear in Math. Ann.

[Sa] M. Sakuma, On regular coverings of links. Math. Ann. 260 (1982), 303-315.

[Su1] M. Suzuki, Group Theory I. Grundlehren der mathematischen Wissenschaften 247. Springer-Verlag, 1982.

[Su2] M. Suzuki, Group Theory II. Grundlehren der mathematischen Wissenschaften 248. Springer-Verlag, 1986.

[T1] W. Thurston, Three dimensional manifolds, Kleinian groups and hyperbolic geometry. Bull. Amer. Math. Soc. 6 (1982), 357-381.

[T2] W. Thurston, 3-manifolds with symmetry. Preprint 1982.

[Z1] B. Zimmermann, On hyperbolic knots with homeomorphic cyclic branched coverings. Math. Ann. 311 (1998), 665-673.

[Z2] B. Zimmermann, On the Hantzche-Wendt manifold. Monatsh. Math. 110 (1990), 321327. 
Bruno Zimmermann

Università degli Studi di Trieste

Dipartimento di Scienze Matematiche

P. le Europa, 1

I-34126 Trieste

Italy

(Received: December 7, 1999; revised version: May 22, 2000) 